\title{
Rate Adaptation in MIMO Antenna Selection System with Imperfect CSIT
}

\author{
T. R. Ramya and Srikrishna Bhashyam \\ Department of Electrical Engineering, \\ IIT Madras, Chennai - 600 036, India. \\ Email: \{ee04d016,skrishna\}@ee.iitm.ac.in
}

\begin{abstract}
In this paper, we analyze the performance of an adaptive Multiple Input Multiple Output (MIMO) system employing transmit antenna selection and rate adaptation based on channel state information (CSI). Imperfections in CSI due to estimation error and feedback delay are considered. Since CSI is imperfect, the rate of transmission is chosen in order to meet a target outage probability. The average throughput is evaluated for various cases of imperfect CSI using Monte Carlo simulations. Simulation results show that rate adaptation provides significant gains even with imperfect CSI. Furthermore, prediction can be used to effectively combat the effect of feedback delay.
\end{abstract}

\section{INTRODUCTION}

Employing adaptive transmission schemes with multiple antenna systems is an effective means to improve the spectral efficiency of wireless communication systems [1], [2]. The major drawbacks are the increased hardware complexity due to the additional antennas and the feedback bandwidth requirements for adaptation. Transmit antenna selection, where the single best antenna is chosen for transmission, is a good low complexity option [3], because of the following reasons: (1) reduced hardware complexity owing to the usage of only one antenna at any point of time, (2) same diversity gain as that of the full system using all the antennas (because diversity gain depends on the total number of antennas rather than the number of antennas that are used), and (3) low feedback bandwith required as the index of the selected antenna alone needs to be fed back.

The performance of a Multiple Input Multiple (MIMO) system employing single transmit antenna selection has been studied in [4] and shown to preserve the diversity gain. Since the wireless channel is time varying in nature, using constant power and rate will lead to block errors during the poor channel conditions or under utilization of resources when the channel conditions are good. Adapting any one of the parameters is sufficient to achieve high gains [5]. Rate adaptation strategies have been shown to provide significant gains both in the case of single antenna systems [6] and multi antenna systems [1]. Adaptive transmission techniques require Channel State Information (CSI) at the transmitter (CSIT). CSI is generally obtained at the receiver using training symbols and fed back to the transmitter through feedback. Estimation errors and feedback delay render the CSIT imperfect. The effect of estimation errors on MIMO antenna selection is studied in [7] and the effect of feedback delay for a specific case of a $2 \times 1$ 978-1-4244-5489-1/10/\$26.00 (c) 2010 IEEE system is studied in [8]. Outage performance and DiversityMultiplexing gain Tradeoff (DMT) of the MIMO antenna selection are presented in [9] for the general imperfect CSIT model that encompasses feedback delay, estimation errors and prediction. However, rate adaptation has not been considered in [7], [8], [9]. In practice, systems with CSIT are likely to adapt the transmission rate while maintaining a target outage probability. Therefore, it is important to understand the effect of imperfect CSIT on a system with rate adaptation along with antenna selection.

In this paper, the performance of a rate adaptive MIMO antenna selection system is studied in the presence of imperfect CSIT. The instantaneous transmission rate is chosen using a lower bound on the conditional mutual information in order to meet a target outage probability. The effect of feedback delay on rate adaptation is studied and the improvement obtained upon using channel prediction is also analyzed. Simulation results reveal that the average throughput improves with rate adaptation even in the presence of feedback delay. However, the improvement reduces with increasing delay. Prediction can effectively combat the impact of feedback delay on adaptive transmission.

The rest of the paper is organized as follows. The system model is described in section II. The lower bound on mutual information and rate adaptation methodology are presented in section III. Simulations results are presented in section IV and conclusions are drawn in section $\mathrm{V}$.

\section{SySTEM MOdEL}

The system model is depicted in Fig 1. A MIMO system with $N_{t}$ transmit antennas and $N_{r}$ receive antennas is considered. At any instant of time, one out of the $N_{t}$ antennas is used for transmission. Transmission rate is adapted based on CSI. The channel between the transmitter and receiver is assumed to be frequency flat. The received vector at time index $k$ is, therefore, represented as:

$$
\mathbf{y}(k)=\sqrt{\bar{P}} \mathbf{h}_{s e l}(k) x(k)+\mathbf{n}(k),
$$

where $x(k)$ represents the transmit symbol at time $k, P$ is the transmit power, $\mathbf{n}(k) \sim \mathcal{C N}\left(0, \sigma_{n}^{2} \mathbf{I}_{N_{r}}\right)$ is the vector of Additive White Gaussian Noise (AWGN) and $\mathbf{h}_{\text {sel }}(k)$ is the $N_{r} \times 1$ channel vector corresponding to the selected transmit antenna. $\mathbf{h}_{\text {sel }}(k)$ is one of the columns of the $N_{r} \times N_{t}$ channel matrix $\mathbf{H}(k)$ at instant $k$. The elements of $\mathbf{H}(k)$ are assumed 


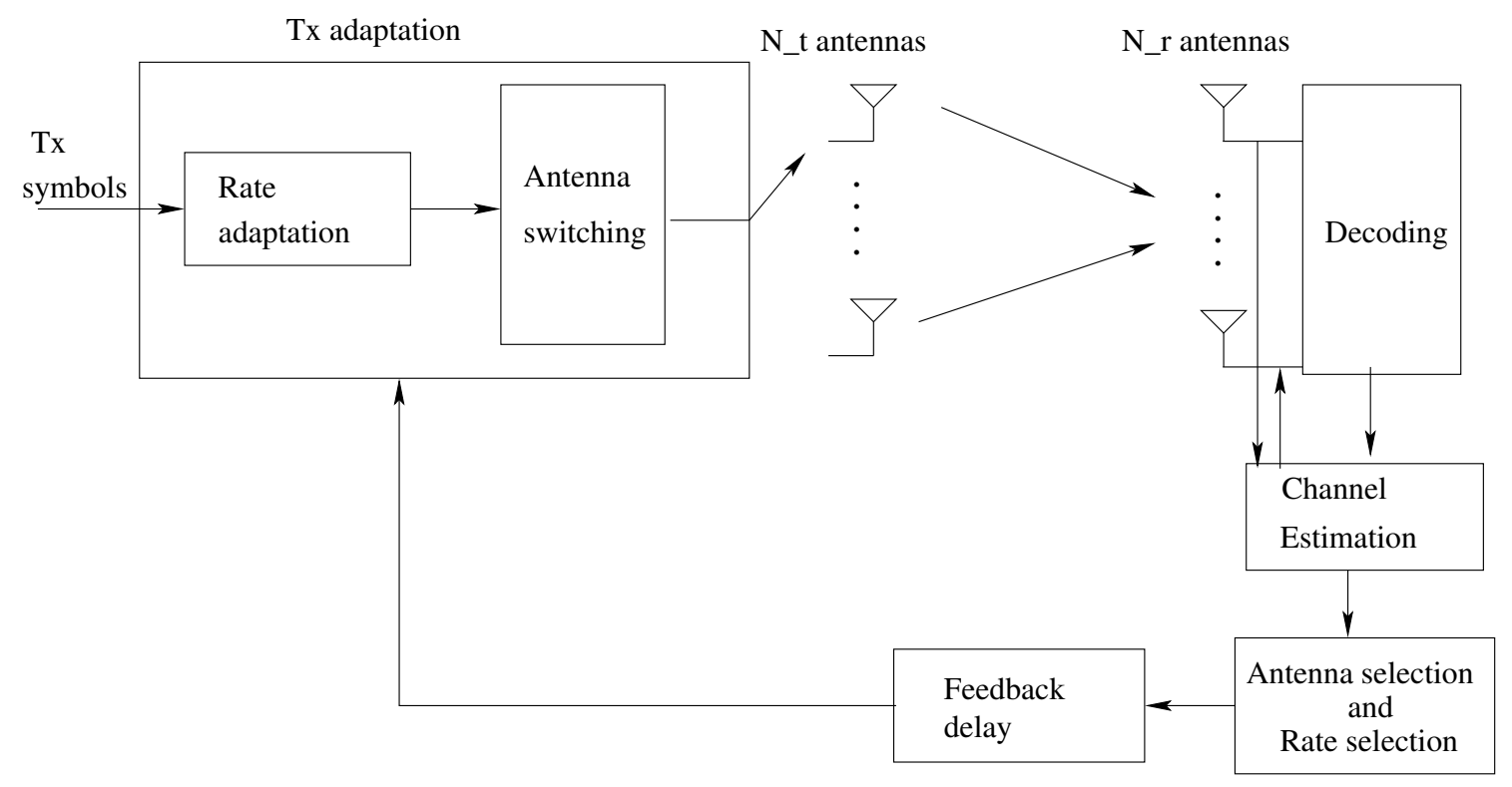

Fig. 1: System Model

to be i.i.d and $\mathcal{C N}(0,1)$. A block fading model is considered, where the elements of $\mathbf{H}$ are assumed to be constant over a block and correlated across blocks.

Maximal Ratio Combining (MRC) is employed at the receiver for decoding. CSI is estimated using training symbols. The training pattern employed for channel estimation is similar to the training in [10], [11]. Antenna selection is done based on the estimated CSI and the selected index is fed back to the transmitter. Selection is based on maximizing the instantaneous receive SNR, i.e., the antenna that maximizes $\left\|\mathbf{h}_{\text {sel }}(k)\right\|^{2}$ is selected. Let $\mathbf{H}_{t}$ represent the channel estimate used for antenna selection and $\mathbf{H}_{r}$ represent the channel estimate used for decoding. Since $\mathbf{H}_{t}$ and $\mathbf{H}_{r}$ are both zero mean and jointly Gaussian and circularly symmetric, they can be related as follows [12], [9]:

$$
\mathbf{H}_{r}=\sigma_{r}\left[\frac{\rho}{\sigma_{t}} \mathbf{H}_{t}+\sqrt{1-\rho^{2}} \mathbf{E}\right],
$$

where $E_{i j} \sim \mathcal{C N}(0,1), \sigma_{r}^{2}=E\left[\left|H_{r, i j}\right|^{2}\right], \sigma_{t}^{2}=E\left[\left|H_{t, i j}\right|^{2}\right]$, and $\rho=\frac{E\left[H_{t, i j} H_{r, i j}^{*}\right]}{\sqrt{E\left[\left|H_{r, i j}\right|^{2}\right] E\left[\left|H_{t, i j}\right|^{2}\right]}} \cdot X_{i j}$ represents the $(i, j)^{t h}$ element of the matrix X. Since $H_{t, i j}$ 's are i.i.d and $H_{r, i j}$ 's are also i.i.d., $\rho$ is independent of $i, j$. We consider four different cases of imperfect CSIT as summarized in Table I. $P_{t}$ is the training symbol power, $f_{d} T_{s}$ is the normalized Doppler frequency, and $\Delta$ is the delay in number of frames. $\mathbf{w}$ is the $L$-tap Wiener filter used for channel prediction and $\mathbf{p}$ is the cross correlation vector between the actual channel and the past estimates. A detailed discussion of the imperfect CSI model can be found in [12], [9].

\section{RATE ADAPTATION FOR THE MIMO ANTENNA SELECTION SYSTEM}

In this section, we present the rate adaptation strategy used with the MIMO antenna selection system. At the time of decoding, the receiver has the knowledge of the current estimate $\mathbf{H}_{r}$ and the past estimate $\mathbf{H}_{t}$. However, antenna selection and rate adaptation have to be performed only using the past estimate $\mathbf{H}_{t}$. When both $\mathbf{H}_{r}$ and $\mathbf{H}_{t}$ are known, a lower bound on the conditional mutual information between the input and output given $\mathbf{H}_{r}$ and $\mathbf{H}_{t}$ can be obtained and a transmission rate can be chosen to avoid outage. However, when only $\mathbf{H}_{t}$ is used to choose the transmission rate, outage is possible. In this case, the transmission rate can be chosen in such a way that the probability that the lower bound on achievable rate is less than the required rate is below a given target outage probability. The details are presented below.

\section{A. Mutual Information Lower bound}

The lower bound on the conditional mutual information between the input $x$ and output $\mathbf{y}$ of the MIMO antenna selection system, for a given $\mathbf{H}_{r}$ and $\mathbf{H}_{t}$ is given by [9]:

$$
I\left(x ; \mathbf{y} / \mathbf{H}_{t}, \mathbf{H}_{r}\right) \geq \log \left(1+\Gamma\left\|\mathbf{H}_{r, s e l}\right\|^{2}\right),
$$

where $\Gamma=\frac{P_{d}}{P_{d} \sigma_{e}^{2}+\sigma_{n}^{2}}, P_{d}$ is the transmit data power and $\mathbf{H}_{r, s e l}$ is the estimate of the channel vector corresponding to the selected antenna. $\sigma_{e}^{2}$ is the estimation error variance given by $\sigma_{e}^{2}=\frac{\sigma_{n}^{2}}{P_{t}+\sigma_{n}^{2}}$. In the absence of estimation errors, i.e., with perfect CSIR, equation (3) becomes an equality.

In practice, $\mathbf{H}_{t}$ alone can be used for rate adaptation. Therefore, this lower bound cannot be calculated. However, since we can relate $\mathbf{H}_{r}$ and $\mathbf{H}_{t}$ using the model in equation (2), we can write

$$
\left\|\mathbf{H}_{r, s e l}\right\|^{2}=\frac{\sigma_{r}^{2}\left(1-\rho^{2}\right)}{2}\left\|\left[\sqrt{\frac{2 \rho^{2}}{\left(1-\rho^{2}\right) \sigma_{t}^{2}}} \mathbf{H}_{t, s e l}+\sqrt{2} \mathbf{E}_{s e l}\right]\right\|^{2}
$$




\begin{tabular}{|c|c|c|c|}
\hline Imperfect CSIT & $\sigma_{r}^{2}$ & $\sigma_{t}^{2}$ & $\rho$ \\
\hline Estimation Errors and no delay & $\frac{P_{t}}{P_{t}+\sigma_{n}^{2}}$ & $\frac{P_{t}}{P_{t}+\sigma_{n}^{2}}$ & 1 \\
\hline No estimation errors, delay & 1 & 1 & $J_{0}\left(2 \pi f_{d} T_{s} \Delta\right)$ \\
\hline Estimation errors + delay & $\frac{P_{t}}{P_{t}+\sigma_{n}^{2}}$ & $\frac{P_{t}}{P_{t}+\sigma_{n}^{2}}$ & $\frac{P_{t}}{P_{t}+\sigma_{n}^{2}} J_{0}\left(2 \pi f_{d} T_{s} \Delta\right)$ \\
\hline $\begin{array}{c}\text { Estimation errors+delay } \\
+ \text { prediction }\end{array}$ & $\frac{P_{t}}{P_{t}+\sigma_{n}^{2}}$ & $\mathbf{p}^{H} \mathbf{w}$ & $\sqrt{\frac{P_{t}}{P_{t}+\sigma_{n}^{2}} \mathbf{p}^{H} \mathbf{W}}$ \\
\hline
\end{tabular}

TABLE I: Imperfect CSIT cases considered

$$
=\sigma_{r}^{2} \frac{\left(1-\rho^{2}\right)}{2} A
$$

where $A$ is defined as $\left\|\left[\sqrt{\frac{2 \rho^{2}}{\left(1-\rho^{2}\right) \sigma_{t}^{2}}} \mathbf{H}_{t, s e l}+\sqrt{2} \mathbf{E}_{s e l}\right]\right\|^{2}$ and $\mathbf{H}_{t, \text { sel }}$ is the vector of past estimates of the selected antenna. Since selection is based on $\mathbf{H}_{t},\left\|\mathbf{H}_{t, \text { sel }}\right\|^{2}=$ $\max _{i=1,2, \cdots, N_{t}}\left\|\mathbf{H}_{t, i}\right\|^{2}$. Therefore, the lower bound on the mutual information for a given $\mathbf{H}_{t}$ and $\mathbf{H}_{r}$ can be written as

$$
I\left(x, \mathbf{y} / \mathbf{H}_{t}, \mathbf{H}_{r}\right) \geq \log \left(1+\Gamma \sigma_{r}^{2} \frac{\left(1-\rho^{2}\right)}{2} A\right) .
$$

The elements of $\sqrt{2} \mathbf{E}_{\text {sel }}$ are complex Gaussian with variance 1 per dimension. Therefore, for a given $\mathbf{H}_{t, \text { sel }}, A$ is noncentral distributed with $2 N_{r}$ degrees of freedom. The non centrality parameter $\delta=\frac{2 \mu}{\sigma_{t}^{2}}\left\|\mathbf{H}_{t, \text { sel }}\right\|^{2}$, where $\mu=\frac{\rho^{2}}{1-\rho^{2}}$. Even though the lower bound in equation (4) cannot be evaluated, the probability that the lower bound is lower than a required rate for a given $\mathbf{H}_{t, s e l}$ can be evaluated. This probability will be an upper bound on the actual outage probability. Therefore, the transmission rate can be chosen such that the outage probability is less than a given target.

For a given $\mathbf{H}_{t}$ and transmission rate $R\left(\mathbf{H}_{t}\right)$, the probability of outage is upper bounded as

$$
\begin{aligned}
& P\left(\text { outage } / \mathbf{H}_{t}\right) \leq P\left(A<2 \beta\left(\frac{1+\mu}{\sigma_{r}^{2}}\right)\right) \\
& =F_{n c-\chi^{2}, 2 N_{r}, \delta}\left(2 \beta\left(\frac{1+\mu}{\sigma_{r}^{2}}\right)\right),
\end{aligned}
$$

where $\beta=\frac{e^{R\left(\mathbf{H}_{t}\right)}-1}{\Gamma}$ and $F_{n c-\chi^{2}, 2 N_{r}, \delta}(\cdot)$ is the CDF of the non central chisquared distributed random variable with parameters $2 N_{r}$ and $\delta$ given by (equation (26.4.25) in [13])

$$
F_{n c-\chi^{2}, 2 N_{r}, \delta}(a)=\sum_{j=0}^{\infty} \frac{e^{-\frac{\delta}{2}}\left(\frac{\delta}{2}\right)^{j}}{j !} \gamma_{j+N_{r}}\left(\frac{a}{2}\right),
$$

where $\gamma_{i}(x)$ is the regularized gamma function given by $\gamma_{i}(x)=\int_{0}^{x} \frac{e^{-y} y^{i-1}}{(i-1) !} d y$.

\section{B. Rate adaptation methodology}

Based on the upper bound on outage probability above, rate adaptation is performed as follows.

Step 1: Find the maximum column norm of $\mathbf{H}_{t}$, i.e., $\left\|\mathbf{H}_{t, \text { sel }}\right\|^{2}$ and the corresponding non-centrality parameter $\delta$.

Step 2: Calculate the largest value of $\beta_{\text {opt }}$ such that

$$
F_{n c-\chi^{2}, 2 N_{r}, \delta}\left(2 \beta_{\text {opt }}\left(\frac{1+\mu}{\sigma_{r}^{2}}\right)\right)<P_{\text {out }},
$$

where $P_{\text {out }}$ is the target outage probability. While it is difficult to get $\beta_{o p t}$ in closed form, standard numerical methods are available to calculate the inverse of the CDF of noncentral chi-squared random variables.

Step 3: Using $\beta_{\text {opt }}$, rate is calculated as

$$
R\left(\mathbf{H}_{t}\right)=\log \left(1+\Gamma \beta_{\text {opt }}\right) .
$$

Finally, the average rate achieved is given by

$$
R_{a v g}=\int_{0}^{\infty} R(x) f_{\left\|\mathbf{H}_{t, s e l}\right\|^{2}}(x) d x,
$$

where $f_{\left\|\mathbf{H}_{t, s e l}\right\|^{2}}(x)$ is the pdf of $\left\|\mathbf{H}_{t, s e l}\right\|^{2}$. This can be evaluated using Monte Carlo simulations.

Some simple special cases where the rate can be chosen to avoid outage are:

1) No delay: In the absence of feedback delay, $\mathbf{H}_{t}=\mathbf{H}_{r}$. In this case, the lower bound on mutual information can be evaluated and, therefore, $R$ can be chosen to be

$$
R\left(\mathbf{H}_{t}\right)=\log \left(1+\Gamma\left\|\mathbf{H}_{r, s e l}\right\|^{2}\right)
$$

in order to avoid outage.

2) Perfect CSIT: With perfect CSIT, $\mathbf{H}_{t}=\mathbf{H}_{r}=\mathbf{H}$ and $\sigma_{e}^{2}=0$. Therefore, $R$ can be chosen as

$$
R(\mathbf{H})=\log \left(1+\frac{P}{\sigma_{n}^{2}}\left\|\mathbf{H}_{s e l}\right\|^{2}\right)
$$

in order to avoid outage.

\section{NUMERICAL RESULTS AND DISCUSSION}

A $2 \times 2$ MIMO antenna selection system is considered for illustration purposes. The average rate (throughput) is calculated using Monte Carlo simulations for the case of perfect CSIT and all the four cases of imperfect CSIT - (1) Estimation errors, no delay, (2) Delay, no estimation errors, (3) Estimation errors, Delay, and (4) Estimation errors, delay, and prediction. The normalized Doppler is chosen to be 0.05 , which leads to $\rho=0.97$. Delay in frames of $\Delta=1$ and $\Delta=2$ are considered. For the case of predicted CSIT, the prediction filter length is chosen to be $L=20$ [12], [9]. Training symbol power is optimized based on the method 
in [11]. The target outage probability $P_{\text {out }}$ is chosen to be 0.01 . The outage capacity without rate adaptation for outage probability of 0.01 is also plotted for the sake of comparison.

Fig. 2 shows the average rate vs. SNR for perfect CSIT and for all the four cases of imperfect CSIT. Feedback delay of $\Delta=1$ frame is considered. It can be seen that rate adaptation improves the average throughput, for all the CSIT conditions. With rate adaptation, even the throughput in the presence of imperfect CSIT is better than that obtained with perfect CSIT in the absence of rate adaptation. Feedback delay affects the rate more significantly compared to estimation error. This is because estimation error reduces with SNR while the feedback delay is independent of SNR. Channel prediction can also be seen to effectively combat delay in feedback.

Fig. 3 compares the effect of using rate adaptation for increasing feedback delay. $\Delta=1$ and $\Delta=2$ are considered. For both the cases, throughput improves upon using rate adaptation. However, the improvement decreases with increasing $\Delta$. As delay increases, the correlation between the available CSIT and the actual CSIT decreases and thereby, the gain achieved using adaptation is also decreased.

The effect of channel prediction is shown in Fig. 4. It is clear from the figure that channel prediction almost restores the gain achieved by adaptation. Using channel prediction improves the correlation between the available CSIT and the actual CSIT. Therefore, the improvement obtained using rate adaptation does not diminish even for increasing feedback delay.

\section{CONCLUSIONS}

In this paper, we have analyzed the performance of rate adaptive MIMO antenna selection systems in the presence of imperfect CSIT. The transmission rate is chosen in order to maintain a target outage probability and different imperfect CSIT cases are considered. It has been shown that rate adaptation improves the average throughput even with imperfect CSIT. However, as delay increases, the gain from rate adaptation decreases. The effect of feedback delay can be significantly compensated using channel prediction. Analytical results on the asymptotic loss in average rate due to imperfect CSIT are being currently studied.

\section{ACKNOWLEDGMENT}

This work was supported in part by the Dept. of Science and Technology, Govt. of India.

\section{REFERENCES}

[1] M. S. Aluoini and A. Goldsmith, "Capacity of Rayleigh fading channels under different adaptive transmission and diversity-combining techniques," IEEE Transactions on Vehicular Technology, vol. 48, no. 4, pp. 1165-1181, Nov. 1999.

[2] S. Jayaweera and H. Poor, "Capacity of multiple-antenna systems with both receiver and transmitter channel state information," IEEE Transactions on Information Theory, vol. 49, no. 10, pp. 2697-2709, Oct. 2003.

[3] A. Molisch, M. Win, Y. Choi, and J. Winters, "Capacity of MIMO systems with antenna selection," IEEE Transactions on Wireless Communications, vol. 4, no. 4, pp. 1759-1772, Jul 2005.

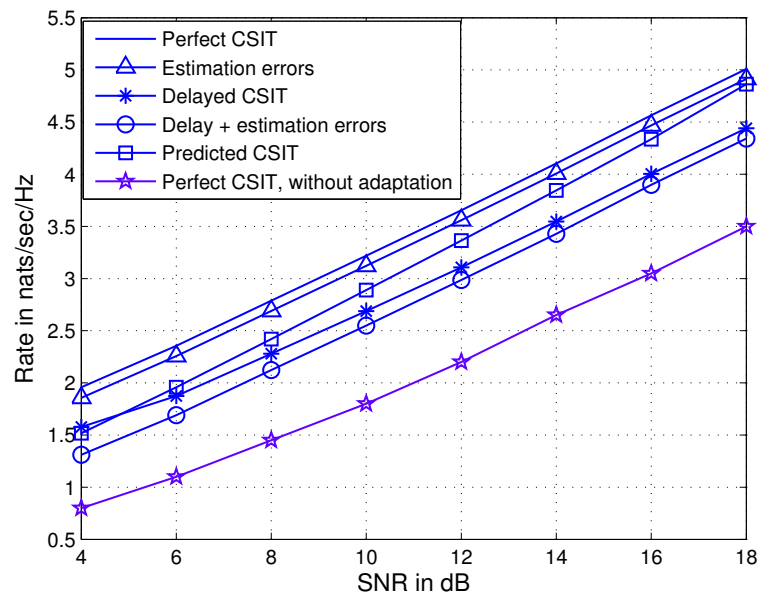

Fig. 2: Average rate vs. SNR for $2 \times 2$ system

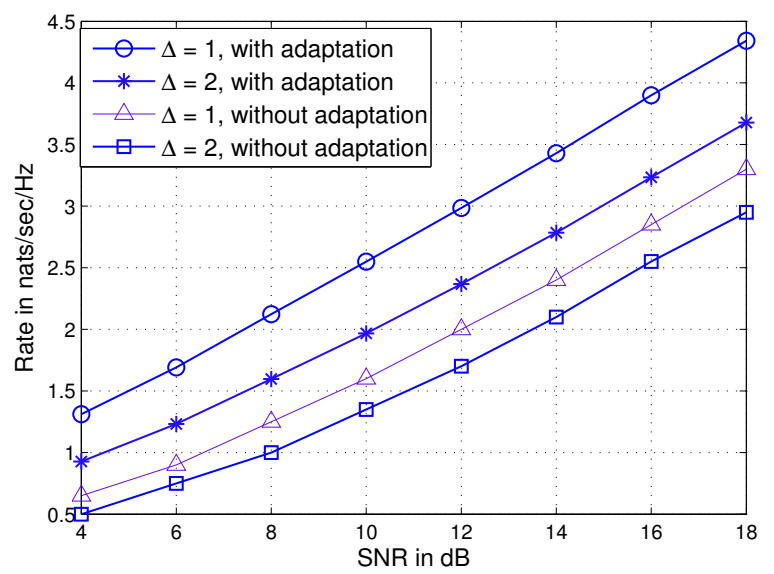

Fig. 3: Average rate vs. SNR for $2 \times 2$ system

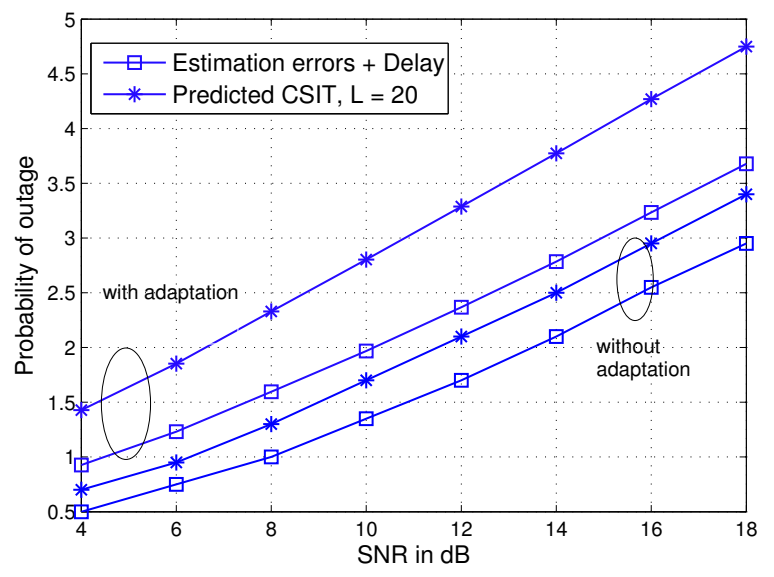

Fig. 4: Average rate vs. SNR for $2 \times 2$ system 
[4] Z. Chen, J. Yuan, and B. Vucetic, "Analysis of transmit antenna selection/maximal-ratio combining in rayleigh fading channels," IEEE Transactions on Vehicular Technology, vol. 54, no. 4, pp. 1312-1321, Jul. 2005.

[5] S. Chung and A. Goldsmith, "Degrees of freedom in adaptive modulation: a unified view," IEEE Transactions on Communications, vol. 49, no. 9, pp. 1561-1571, Sep. 2001.

[6] A. Goldsmith and S. Chua, "Variable-rate variable-power MQAM for fading channels," IEEE Transactions on Communications, vol. 45, no. 10, pp. 1218-1230, Oct. 1997.

[7] W. Gifford, M. Win, and M. Chiani, "Antenna Subset Diversity with Non-Ideal Channel Estimation," IEEE Transactions on Wireless Communications, vol. 7, no. 5, pp. 1527-1539, May 2008.

[8] S. Han and C. Yang, "Performance analysis of MRT and transmit antenna selection with feedback delay and channel estimation error," in IEEE Wireless Communications and Networking Conference, 2007. WCNC 2007, Mar. 2007, pp. 1134-1138.

[9] T. R. Ramya and S. Bhashyam, "Using delayed feedback for antenna selection in mimo systems," To appear in IEEE Transactions on Wireless Communications, 2009, http://www.ee.iitm.ac.in/ skrishna/publications.html.

[10] B. Hassibi and B. Hochwald, "How much training is needed in multipleantenna wireless links?" IEEE Transactions on Information Theory, vol. 49, no. 4, pp. 951-963, April 2003.

[11] S. Bhashyam, A. Sabharwal, and B. Aazhang, "Feedback gain in multiple antenna systems," IEEE Transactions on Communications, vol. 50, no. 5, pp. 785-798, May 2002.

[12] T. R. Ramya and S. Bhashyam, "Eigen-beamforming with delayed feedback and channel prediction," in Proceedings of IEEE ISIT, Seoul, Korea, Jun-Jul. 2009, pp. 403-407.

[13] M. Abramowitz and I. A. Stegun, Handbook of Mathematical Functions. Dover Publications (New York), 1964 